\title{
Volume Curve
}

National Cancer Institute

\section{Source}

National Cancer Institute. Volume Curve. NCI Thesaurus. Code C95016.

Plot of a ventricle volume as the ordinate vs. time in a cardiac cycle as the abscissa. 